\title{
GRUPO FOCAL COMO TÉCNICA DE COLETA DE DADOS
}

Josefine Busanello, Wilson Danilo Lunardi Filho², Nalú Pereira da Costa Kerber ${ }^{3}$, Silvana Sidney Costa Santos², Valéria Lerch Lunardi², Flávia Conceição Pohlmann ${ }^{4}$

RESUMO: Revisão integrativa que objetivou identificar características metodológicas empregadas nas publicações científicas da Enfermagem que utilizaram o Grupo Focal como técnica de coleta de dados. O levantamento bibliográfico foi realizado em novembro de 2010, nas bases de dados da Biblioteca Virtual em Saúde, utilizando os descritores Grupos focais e Enfermagem. Foram selecionados 55 artigos, com os principais resultados: publicações a partir 2002; temáticas tangenciaram, principalmente, o Ensino e Pesquisa da Enfermagem; número de encontros focais variou de um a 20; média de participantes foi 10; mais de $32 \%$ dos estudos utilizaram análise temática. Este estudo poderá apoiar a construção de estudos que utilizem essa técnica de coleta de dados, considerando que o grupo focal favorece a percepção de práticas, ações e reações a eventos, comportamentos e atitudes que influenciam na saúde ou doença.

DESCRITORES: Enfermagem; Pesquisa qualitativa; Pesquisa em enfermagem; Grupos focais.

\section{THE FOCUS GROUP AS A TECHNIQUE FOR DATA COLLECTION}

ABSTRACT: This integrative review aimed to identify methodological characteristics used in Nursing scientific productions which used the Focus Group as a technique for data collection. The literature survey was undertaken in November 2010, in the databases of the Virtual Health Library, using the descriptors Focus Groups and Nursing. A total of 55 articles was selected, with the main results: publications since 2002; topics which were to do with, principally, Teaching and Research in Nursing; the number of focus group meetings varied between one and 20; the average number of participants was 10; more than $32 \%$ of the studies used thematic analysis. This study will support the construction of studies which use this data collection technique, taking into account that the focus group favors the perception of practices, actions and reactions to events, and behaviors and attitudes which influence health or illness.

DESCRIPTORES: Nursing; Qualitative research; Nursing research; Focus groups.

\section{GRUPO FOCAL COMO TÉCNICA PARA OBTENER DATOS}

RESUMEN: Revisión integrativa que tuvo el objetivo de identificar características metodológicas utilizadas en las publicaciones científicas de la enfermería cuya técnica para obtener datos fue el Grupo Focal. La investigación bibliográfica fue realizada en noviembre de 2010, en las bases de datos de la Biblioteca Virtual en Salud, utilizando los descriptores Grupos focales y Enfermería. Fueron seleccionados 55 artículos, con los principales resultados: publicaciones a partir de 2002; temáticas acerca de, principalmente, la Enseñanza e investigación de la enfermería; número de encuentros focales varió de un a 20; media de participantes fue 10; más de 32\% de los estudios utilizaron análisis temático. Este estudio podrá apoyar la construcción de trabajos que utilizen esa técnica para recoger datos, considerando que el grupo focal favorece la percepción de prácticas, acciones y reacciones a eventos, comportamientos y actitudes que influencian en la salud o enfermedad. DESCRIPTORES: Enfermería; Investigación cualitativa; Investigación en enfermería; Grupos focales.

\footnotetext{
${ }^{1}$ Enfermeira. Doutora em Enfermagem. Professora do Curso de Graduação em Enfermagem da Universidade Federal do Pampa. ${ }^{2}$ Enfermeiro. Doutor em Enfermagem. Professor da Escola de Enfermagem da FURG. Pesquisador do CNPq.

${ }^{3}$ Enfermeira. Doutora em Enfermagem. Professora da Escola de Enfermagem da FURG.

${ }^{4}$ Acadêmica de Enfermagem da FURG. Bolsista PIBIC-CNPq.
} 


\section{INTRODUÇÃO}

A grande parte das investigações científicas, que tem como objetivo avaliar os programas e ações de saúde implementadas no âmbito internacional, tem sido desenvolvidas a partir de uma abordagem qualitativa ${ }^{(1)}$. Esse tipo de pesquisa tende a ser a primeira opção, quando se sabe pouco a respeito de um determinado fenômeno, experiência ou conceito, e busca evidenciar a evolução das ideias, a partir da descrição e interpretação de fenômenos passíveis de investigação, utilizando como matéria a experiência humana ${ }^{(2)}$.

O grupo focal é considerado uma técnica de coleta de dados específico das pesquisas com abordagem qualitativa, por proporcionar a interação grupal para a produção de dados que seriam menos acessíveis fora do contexto interacional. A partir dessa técnica, é possível coletar dados, diretamente, dos depoimentos de um grupo, que relata suas experiências e percepções, em torno de um tema de interesse coletivo ${ }^{(3)}$.

No âmbito científico da Enfermagem, os grupos focais são utilizados como técnicas de coleta de dados por favorecerem a exploração de temas que necessitam de interação efetiva com a população. Além disso, essa técnica permite compreender processos de construção da realidade por determinados grupos sociais e entender práticas cotidianas, ações e reações a fatos e eventos, comportamentos e atitudes que, direta ou indiretamente, influenciam na saúde e na doença ${ }^{(4)}$.

Considerando o interesse e utilização crescente da técnica grupo focal para a coleta de dados em pesquisas científicas da Enfermagem, o presente estudo busca responder à seguinte pergunta: Quais foram as características metodológicas empregadas nas publicações científicas da Enfermagem que utilizaram o grupo focal como técnica de coleta de dados?

Para a implementação dos encontros focais, alguns aspectos relevantes precisam ser considerados nos desenhos metodológicos dos estudos. As discussões são grupais, devendo ser fomentadas por uma série de perguntas abertas, estimulantes e suficientes para explorar a temática em estudo; a composição do grupo deve ser modesta com, no mínimo, quatro e, no máximo, oito pessoas; a seleção da amostra precisa ser intencional ou teoricamente planejada, apresentando homogeneidade entre os participantes; o desenvolvimento dos grupos focais precisa ocorrer em um ambiente agradável e confortável para os participantes, não ultrapassando o período de uma a duas horas; o pesquisador deve atuar como facilitador do grupo, explicando o objetivo principal do estudo, favorecendo e estimulando as discussões ${ }^{(5)}$.

Diante desses aspectos que condicionam a utilização da técnica de coleta de dados em questão, a presente revisão integrativa tem como objetivo identificar características metodológicas empregadas nas publicações científicas da Enfermagem que utilizaram o frupo focal como técnica de coleta de dados.

\section{MÉTODO}

Trata-se de uma revisão integrativa, método que tem como finalidade a sumarização de pesquisas já finalizadas para obter novas conclusões, a partir de um tema de interesse. Na operacionalização dessa revisão, utilizaram-se as seguintes etapas: definição da questão norteadora; busca dos estudos; seleção dos estudos; avaliação criteriosa dos estudos; coleta de dados; e síntese e análise dos dados ${ }^{(6-7)}$.

Após a definição da questão norteadora prosseguiu-se com a etapa de levantamento bibliográfico, realizado em novembro de 2010, nas seguintes bases de dados da Biblioteca Virtual em Saúde: Literatura Latino-Americana e do Caribe em Ciências da Saúde (LILACS); Índice Bibliográfico Espanhol de Ciência de Saúde (IBECS); Literatura Internacional em Ciência da Saúde (MEDLINE); Biblioteca Cochrane; e Scientific Electronic Library Online (SciELO). Optou-se por essas bases de dados, considerando que nestas estão indexadas as principais produções científicas da Enfermagem.

Foram utilizados os descritores 'Grupos focais e Enfermagem', com busca livre e sem delimitação do período de publicação. Os critérios para inclusão dos estudos na presente revisão foram: artigos publicados em português, espanhol ou inglês; disponíveis em texto completo; e que utilizaram o grupo focal como técnica de coleta de dados.

Posteriormente a essa seleção, foi realizada avaliação criteriosa dos estudos, mediante a leitura exploratória do texto completo dos artigos científicos, com intuito de coletar dados, a partir de um instrumento que buscou identificar características metodológicas acerca da técnica de grupo focal: ano de publicação; temática; método de coleta dos dados; número de encontros; tempo de encontro; número de participantes; método de análise dos dados; e aspectos relevantes da utilização do grupo focal como técnica de coleta de dados. 
Para síntese dos dados, foram utilizados dois métodos: a distribuição de frequência e a análise descritiva, a partir do software SPSS - Statistical Package for the Social Sciences. Também foi usada a análise qualitativa ${ }^{(8)}$, desenvolvida por meio da leitura analítica, seguindo as etapas de análise textual, realizada a partir da leitura cuidadosa, obtendo-se uma visão mais abrangente dos dados; análise temática, buscando esclarecimentos a respeito do tema abordado; e análise interpretativa, na qual se desenrolou a problematização dos achados.

\section{RESULTADOS}

Inicialmente, foram encontrados 126 estudos no levantamento bibliográfico inicial. Considerando os critérios de seleção adotados, foram desconsiderados: 56 estudos que não disponibilizavam texto completo; dois estudos que não utilizavam o grupo focal como técnica de coleta de dados; um estudo do tipo revisão integrativa; sete dissertações; e cinco teses.

Os periódicos científicos em que foram publicados os artigos, em sua maioria, são da Enfermagem, com destaque para a Revista da Escola de Enfermagem da Universidade de São Paulo e a Revista Acta Paulista de Enfermagem. Outros periódicos, também específicos da área, apresentaram a publicação de estudos que utilizaram essa técnica de coleta de dados: Revista Texto e Contexto - Enfermagem, Revista Brasileira de Enfermagem e Cogitare Enfermagem.

Assim, a presente revisão é composta por 55 artigos científicos. De acordo com a tabela 1, observa-se que a produção científica analisada concentra-se no período compreendido entre os anos de 2002 a 2010. O ano de 2008 apresentou o maior número de publicação.

Os estudos da Enfermagem que utilizaram o grupo focal abordaram, em sua maioria, temáticas associadas ao Ensino e Pesquisa da Enfermagem, como pode ser observado na tabela 2. Outras temáticas que apresentaram um número significativo de estudos foram a Saúde Coletiva e o Processo de Trabalho da Enfermagem.

Conforme a tabela 3, evidencia-se que mais de $40 \%$ dos estudos utilizaram somente o grupo focal como técnica de coleta de dados. No entanto, $15 \%$ dos estudos analisados, além de utilizar essa técnica, associaram outras técnicas de coleta de dados, tais como entrevista, observação; e análise documental; e entrevista e observação.

Em torno de $18 \%$ dos estudos analisados não informaram o número de participantes dos encontros focais. Conforme os estudos que informaram este dado, é possível destacar que mais de $16 \%$ dos estudos tiveram 9 participantes; o mínimo de participantes foi quatro e o máximo foi 20; e a média de participantes foi, aproximadamente, 10.

De acordo com tabela 4, observa-se que mais de $60 \%$ dos estudos analisados utilizaram a técnica de análise de conteúdo para análise dos dados. Outras técnicas adotadas, em menor frequência foram análise do discurso, discurso do sujeito coletivo e Ground Theory.

A partir da presente revisão, evidencia-se que o número de encontros, realizados nos estudos da Enfermagem que utilizaram o grupo focal, variam de um a 20 , e mais de $40 \%$ dos estudos analisados não disponibilizavam essa informação. Nos artigos que descreveram essa característica metodológica, identificou-se que a maioria realizou apenas um encontro, enquanto que a média de encontros focais foi de três a quatro.

Em relação ao tempo de duração dos encontros dos focais, observa-se que mais de $60 \%$ dos estudos analisados não disponibilizaram essa informação. Dentre os artigos que descreveram esse aspecto, evidencia-se que a maioria teve a duração de uma hora e trinta minutos; a média de tempo ficou entre uma hora e trinta minutos e duas horas; o mínimo de duração das sessões focais foi de uma hora, e o máximo, de três horas.

Das 55 publicações científicas analisadas, apenas quatro estudos apresentaram as implicações da utilização do grupo focal. Dentre os aspectos positivos acerca dessa técnica de coleta de dados, estas pesquisas destacaram a possibilidade de interação entre o âmbito acadêmico e o campo assistencial, o que favorece a criação de espaços para o fortalecimento da Enfermagem.

Ao investigar as recomendações propostas pelos estudos, acerca do processo metodológico que envolve o grupo focal, evidenciou-se a escassez de dados desta natureza. Contudo, foram identificadas contribuições importantes dessa técnica de coleta de dados para as pesquisas analisadas: aproximação entre os participantes (participantes e pesquisadores); troca de saberes acerca do fenômeno em estudo; e estabelecimento de um espaço para reflexão e discussão entre os envolvidos. Essas perspectivas configuram o grupo focal como uma técnica de coleta de dados diferenciada, pois favorecem a interação grupal para a busca de dados que seriam menos acessíveis fora do contexto coletivo. 
Tabela 1 - Distribuição dos estudos de acordo com o ano de publicação. Rio Grande, 2010

\begin{tabular}{lcc}
\hline Período de publicação & Frequência & $\mathbf{\%}$ \\
\hline 2002 a 2006 & 17 & 31 \\
2007 & 10 & 18,2 \\
2008 & 13 & 23,6 \\
2009 & 11 & 20 \\
2010 & 4 & 7,3 \\
\hline Total & 55 & 100 \\
\hline
\end{tabular}

Tabela 2 - Distribuição dos estudos de acordo com a temática abordada. Rio Grande, 2010

\begin{tabular}{lcc}
\hline Área Temática & Frequência & \% \\
\hline Saúde Materno Infantil & 4 & 7,3 \\
Saúde Coletiva & 7 & 12,7 \\
Gerenciamento em Saúde e & 4 & 7,3 \\
$\begin{array}{l}\text { Enfermagem } \\
\text { Ensino e Pesquisa em }\end{array}$ & 22 & 40 \\
$\begin{array}{l}\text { Enfermagem } \\
\text { Processo de Trabalho da }\end{array}$ & 8 & 14,5 \\
Enfermagem & 10 & 18,2 \\
\hline Outras temáticas & 55 & 100 \\
\hline Total & & \\
\hline
\end{tabular}

Tabela 3 - Distribuição dos estudos de acordo com a técnica de coleta de dados. Rio Grande, 2010

\begin{tabular}{lcc}
\hline Técnica de coleta de dados & Frequência & $\mathbf{\%}$ \\
\hline Grupo focal e entrevista & 12 & 21,8 \\
$\begin{array}{l}\text { Grupo focal e observação } \\
\text { Grupo focal e análise de }\end{array}$ & 1 & 1,8 \\
$\begin{array}{l}\text { documentos } \\
\begin{array}{l}\text { Grupo focal, entrevista e } \\
\text { observação }\end{array}\end{array}$ & 2 & 3,6 \\
$\begin{array}{l}\text { Grupo focal, entrevista e } \\
\text { análise de documentos }\end{array}$ & 36 & 65,3 \\
\hline Total & 55 & 100 \\
\hline
\end{tabular}

Tabela 4 - Distribuição dos estudos de acordo com a técnica de análise de dados. Rio Grande, 2010

\begin{tabular}{lcc}
\hline Técnica de análise de dados & Frequência & $\mathbf{\%}$ \\
\hline Não informou & 5 & 9,1 \\
Análise de Conteúdo & 35 & 63,6 \\
Análise do Discurso & 8 & 14,5 \\
Discurso de Sujeito Coletivo & 6 & 10,9 \\
Ground Theory & 1 & 1,8 \\
\hline Total & 55 & 100 \\
\hline
\end{tabular}

\section{DISCUSSÃO}

Observa-se que a utilização do grupo focal, no âmbito científico da Enfermagem, é recente, assim como em outras áreas. Somente a partir de 2002 essa técnica de coleta de dados passou a ser explorada pelos pesquisadores enfermeiros. No Brasil, os primeiros estudos desenvolvidos, a partir dessa técnica de coleta de dados, foram na Faculdade de Saúde Pública da Universidade Federal de São Paulo, na área de Educação em Saúde, desde 1989, com a finalidade de diagnosticar problemas educativos em saúde e em estudos avaliativos de programas de saúde ${ }^{(1)}$.

A técnica de grupo focal aparece em estudos publicados nos principais periódicos científicos nacionais da Enfermagem. Nesse sentido, observou-se que essa técnica conquistou significado importante entre os enfermeiros brasileiros, nas mais diferentes áreas do conhecimento da Enfermagem. Em especial, no âmbito do ensino e pesquisa, por proporcionar uma visão coletiva do objeto de estudo, pois favorece a obtenção de perspectivas diferentes sobre uma mesma questão, e torna oportuna a compreensão de ideias coletivas, que são elaboradas a partir da influência que o convívio social proporciona ${ }^{(4)}$.

Outra característica metodológica observada, a partir da revisão integrativa, foi a forte tendência da associação do grupo focal com outras técnicas de coleta de dados. Essa perspectiva não indica a insuficiência dessa técnica. Na tentativa de aumentar a validade das pesquisas qualitativas, os investigadores têm explorado a triangulação, que consiste em utilizar duas ou mais teorias, métodos, fontes de dados, investigadores (pesquisadores) ou métodos de análise em um mesmo estudo, buscando obter diversos pontos de vista do fenômeno sob estudo com propósitos de validação ${ }^{(9)}$.

Considerando a operacionalização do grupo focal, evidenciou-se que, a grande maioria dos estudos utiliza três a quatro encontros, perspectiva condizente com os referências teóricos ${ }^{(4,10)}$. A determinação do número de sessões varia de acordo com o universo temático pesquisado $^{(10)}$, sendo que não há padrão rígido para definir essa característica metodológica, podendo ser definida ao longo da pesquisa. Em alguns estudos, um encontro é suficiente para que as discussões sejam consolidadas. Em outros, há necessidade de um maior número de sessões focais, para que os objetivos propostos pelo estudo sejam alcançados ${ }^{(4)}$.

Constatou-se que os estudos desenvolvidos pela Enfermagem desenvolveram as sessões em período 
de uma hora e trinta minutos a duas horas, tempo sugerido por referenciais metodológicos ${ }^{(5,10)}$. Além do tempo dedicado às discussões do grupo, é preciso considerar a necessidade de um período de organização inicial da sessão, que inclui a interação entre os participantes, e um espaço para o encerramento do encontro. Em encontros focais que ultrapassam duas horas pode ocorrer fadiga entre os participantes ou intelectualizações excessivas acerca do tema, em virtude do desgaste ou esvaziamento da mobilização do grupo $^{(10)}$.

A dimensão de participantes do grupo deve variar de acordo com os objetivos e finalidades estabelecidas. Um grupo maior, de até 15 participantes, pode ser indicado nos estudos que buscam gerar o máximo de discussões e ideias possíveis em torno de uma temática $^{(10)}$. Tradicionalmente, o grupo focal é composto, em média, por 10 participantes, e a seleção destes deve preservar a homogeneidade entre os mesmos ${ }^{(1,10)}$.

Essa perspectiva, relacionada à dimensão do grupo, foi evidenciada na maioria dos estudos analisados. Contudo, dimensões menores, entre 5 e 7 participantes, são utilizados em estudos com a finalidade de maximizar a profundidade de expressões de cada participante ${ }^{(10)}$.

Além dos participantes, o grupo focal é composto por um moderador que, em geral, é o pesquisador e tem como missão promover a interação dos participantes, fomentando as discussões e expressões. Observadores também podem ser incluídos, com a função de avaliar a condução técnica dos encontros, organizando o ambiente, fazendo o registro dos depoimentos e observando a dinâmica do grupo $^{(4)}$. O observador, em geral, deve estar envolvido com o tema em questão, podendo atuar a partir da observação participante ou não participante, de acordo com o caminho metodológico adotado pelo pesquisador responsável pelo estudo ${ }^{(10)}$.

Alguns dados importantes não foram identificados nos estudos analisados, tais como a avaliação dos autores acerca da utilização do grupo focal, e as possíveis recomendações metodológicas acerca dessa técnica de coleta de dados. De um modo geral, é relevante a apresentação dessas informações nas etapas finais dos estudos, especificamente, na elaboração das considerações finais e conclusões.

Em sua maioria, os artigos não apresentam parecer acerca da metodologia utilizada, omitindo um dado importante em relação ao processo metodológico adotado. Possivelmente, se a revisão integrativa fosse conduzida nos documentos originais das pesquisas analisadas, essas informações poderiam ser observadas, pois os periódicos determinam o número de páginas para a estruturação dos artigos científicos, reduzindo as informações metodológicas das pesquisas.

De um modo geral, o desenvolvimento da parte final de uma pesquisa ou publicação científica tem um tempo reduzido, em relação às demais etapas de um estudo. No fechamento de um manuscrito, o pesquisador precisa articular os resultados mais relevantes e significativos, procurando demonstrar a aplicabilidade, praticidade e possibilidade de ampliação do conhecimento produzido. Além disso, o capítulo final do estudo, por vezes, pode ser utilizado como um espaço para a auto avaliação do investigador, apontando caminhos metodológicos que podem ter fragilizado ou prejudicado o andamento da pesquisa ${ }^{(11)}$.

A partir da revisão, também foram identificadas as contribuições do grupo focal. De acordo com um estudo que abordou a compreensão dos efeitos da função renal e sua relação com a hipertensão, essa técnica de coleta de dados possibilitou a interação, permitindo uma troca recíproca de saberes acerca do fenômeno em estudo. Ademais, essa técnica de coleta de dado favoreceu a manifestação dos participantes que revelaram o contexto e a realidade de $\operatorname{vida}^{(12)}$. Essa interação de igual forma foi evidenciada por uma investigação que utilizou o grupo focal para descrever a percepção de enfermeiras acerca dos elementos do seu processo de trabalho em Central de Material e Esterilização. Neste estudo a técnica permitiu uma discussão produtiva pautada na troca de experiências, opiniões, exposição de ideias e reflexões ${ }^{(13)}$.

Um estudo realizado em um hospital de grande porte da Região Sul do Rio Grande do Sul, que teve como objetivo promover a reflexão de enfermeiras sobre seu processo de trabalho, destacou que a utilização do grupo focal proporcionou às enfermeiras, juntamente com as pesquisadoras, momentos de reflexão, os quais futuramente poderão contribuir para transformações concretas nessa instituição hospitalar. Essa técnica permitiu o estabelecimento de um espaço de discussão e reflexão no qual as participantes foram incentivadas a assumir, conscientemente, sua posição de atores sociais, bem como de seres políticos, críticos, reflexivos, capazes de utilizar de forma coerente a ação e o discurso, a fim de transformar a realidade em que estão inseridas ${ }^{(14)}$.

O grupo focal favorece a oportunidade de autoconhecimento, de autoafirmação, de revisão conceitual e de reflexão crítica, acerca das atividades cotidianas. Essa perspectiva reforça uma das vantagens dessa técnica de coleta de dados que é a de conduzir os par- 
ticipantes ao pensamento crítico. Assim, essa técnica favorece a percepção dos significados que expressam o ponto de vista de quem foi pesquisado, singularidades, experiência, vivências e sentimentos ${ }^{(15)}$.

As publicações científicas analisadas não apontaram fragilidades ou aspectos negativos acerca do uso do grupo focal. No entanto, cabe ressaltar que nem sempre essa técnica é adequada para todos os estudos. De um modo geral, pode ser mais bem explorada como técnica de coleta de dados em pesquisas qualitativas, pois permite em pouco tempo e baixo custo, investigar questões complexas e produzir conhecimento ${ }^{(16)}$. Todavia, a escolha do desenho metodológico precisa corresponder ao tema e ao objeto de estudo. Por exemplo, temas que envolvem a intimidade do paciente e de sua família, enfocados em um grupo, podem constranger os participantes, prejudicando as discussões ${ }^{(1)}$.

O grupo focal, assim como as demais técnicas de coleta de dados, requer um desenho metodológico bem definido, pois a organização e o planejamento da pesquisa tem impacto direto no resultado dos dados coletados ${ }^{(17)}$. Assim, ao definir o método da pesquisa é importante considerar a adequação entre o tipo de abordagem e a técnica de coleta de dados, ao objeto e ao contexto em estudo, garantindo a significância do conhecimento produzido.

\section{CONSIDERAÇÕES}

A partir do presente estudo, foi possível identificar algumas características metodológicas das publicações científicas da Enfermagem que utilizaram o grupo focal como técnica de coleta de dados. Todavia, destaca-se que o desenho metodológico dos estudos não foi analisado completamente, pois o tipo de estudo, o periódico, as informações acerca dos autores, e outros aspectos, não foram explorados, constituindo uma limitação desta revisão integrativa.

Nas pesquisas científicas, a escolha da técnica de coleta de dados, parte do objetivo proposto e da possibilidade de investigação do fenômeno. Porém, mesmo utilizando um referencial metodológico, as técnicas podem ser adaptadas ao ambiente, aos participantes e ao contexto em estudo. A variação do número e o tempo de duração dos encontros e de participantes, evidenciada entre os estudos analisados, pode representar essa necessidade de adaptação.

Em relação ao método de revisão integrativa utilizada no presente estudo, salienta-se que o mesmo, de um modo geral, vem sendo amplamente utilizado com o objetivo de mapear o conhecimento produzido, acerca de um determinado tema. No entanto, enfatiza-se que esse método também pode ser utilizado como um meio de visualizar os desenhos metodológicos e as especificidades de determinadas técnicas. Um dos aspectos negativos do estudo foi o fato de que nem todas as publicações científicas estão disponíveis em texto completo nas bases de dados e, para garantir o acesso na totalidade, o custo de uma revisão integrativa pode se tornar elevado.

Neste sentido, entende-se que a presente revisão pode ter aplicabilidade e praticidade, em especial, no que se refere à finalidade a que foi idealizada. Ademais, os dados apresentados poderão servir de apoio para a construção de outros estudos que pretendem utilizar essa técnica de coleta de dados. Nas pesquisas na área da Enfermagem, o grupo focal favorece a percepção de práticas cotidianas, ações e reações a fatos e eventos, comportamentos e atitudes que, direta ou indiretamente, influenciam na saúde e na doença. Essas perspectivas podem aproximar os enfermeiros do principal objeto de estudo da Enfermagem: o cuidado ao ser humano em situações de saúde e de doença.

\section{REFERÊNCIAS}

1. Lervolino SA, Pelicioni MCF. A utilização do grupo focal como metodologia qualitativa na promoção da saúde. Rev Esc Enferm USP. 2001;35(20):115-21.

2. Bruggemann OM, Parpinelli MA. Utilizando as abordagens quantitativa e qualitativa na produção do conhecimento. Rev Esc Enferm USP. 2008;42(3):563-8.

3. Leopardi MT, Beck CLC, Nietsche EA, Gonzales RMB. Metodologia da pesquisa na saúde. Santa Maria: Pallotti; 2001.

4. Gomes VLO, Telles KS, Roballo EC. Grupo focal e discurso do sujeito coletivo: produção de conhecimento em saúde de adolescentes. Esc. Anna Nery. 2009;13(4):856-62.

5. Kitzinger J. Grupos focais com usuários e profissionais da atenção à saúde. In: Pope C, Mays N. Pesquisa qualitativa na atenção à saúde. Porto Alegre: Artmed; 2009. p. 33-43.

6. Whittemore R, Knafl K. The integrative review: updated methodology. J Adv Nurs. 2005;52(5):546-53.

7. Mendes KDS, Silveira RCCP, Galvão CM. Revisão 
integrativa: método de pesquisa para a incorporação de evidências na saúde e na enfermagem. Texto Contexto Enferm. 2008;17(4):758-64.

8. Severino AJ. Metodologia do trabalho científico. São Paulo: Cortez; 2002.

9. Burns N, Grove SK. The practice of nursing research: conduct, critique \& utilization. United States of American: Saubbders; 2001.

10. Dall'Agnol CM, Trench MH. Grupos focais como estratégia metodológica em pesquisas na enfermagem. Rev. Gaúcha Enferm. 1999;20(1):5-25.

11. Becker PT. Thoughts on the end of the article: the implications for nursing practice. Res Nurs Health. 2009;(32):241-2.

12. Orsolin C, Rufatto C, Zambonato RX, Fortes VL, Pomati DM. Cuidando do ser humano hipertenso e protegendo sua função renal. Rev Bras Enferm. 2005;58(3):316-19.

13. Taube SAM, Meier MJ. O processo de trabalho da enfermeira na central de material e esterilização. Acta Paul. Enferm. 2007;20(4):470-5.

14. Amestoy SC, Cestari ME, Thofehrn MB, Milbrath VM, Porto AR. Enfermeiras refletindo sobre seu processo de trabalho. Cogitare enferm. 2010;15(1):158-63.

15. Ressel LB, Beck CLC, Gualda DMR, Hoffmann IC, Silva RM, Sehnem GD. O uso do grupo focal em pesquisa qualitativa. Texto Contexto Enferm. 2008;17(4):779-86.

16. Aschidamini IM, Saupe R. Grupo focal estratégia metodológica qualitativa: um ensaio teórico. Cogitare enferm. 2004;9(1):9-14.

17. Mazza VA, Melo NSFO, Chiesa AM. O grupo focal como técnica de coleta de dados na pesquisa qualitativa: relato de experiência. Cogitare enferm. 2009;14(1):183-8. 